\title{
Bei Proktitis auch an Chlamydien und Gonokokken denken!
}

Eine Proktitis lässt zunächst einmal an eine chronisch entzündliche Darmerkrankung denken. Sich vorschnell festzulegen, ist allerdings ein Fehler, denn es kommt eine ganze Reihe von Differenzialdiagnosen in Betracht. Dazu gehören auch sexuell übertragene Erkrankungen.

Steckt hinter einer Proktitis eine Colitis ulcerosa ist, ist meist eine lokale Therapie mit Mesalazin (z.B. Salofalk ${ }^{\circledR}$ ) oder auch Budesonid (z.B. Budenofalk ${ }^{\circ}$ ) erfolgreich, so PD Dr. Nils Teich, Leipzig. Spricht die Entzündung darauf nicht an, muss auch eine sexuell übertragene infektiöse Gene- se in Erwägung gezogen werden. Immerhin finden sich bei $11 \%$ der asymptomatischen homosexuellen Männer Chlamydien oder Gonokokken im Rektumabstrich, erläuterte Teich. Was makroskopisch wie eine Proktitis ulcerosa daherkommt, kann also auch die Manifestation eines Lymphogranuloma venerum (LVG) sein. In Betracht kommen als Ursache der Proktitis auch Gonorrhö, Herpes oder gar eine Syphilis. Wegweisend sind die Begleitsymptome. Beim LVG treten oft asymptomatische genitale und perianale Ulzerationen, Fieber und Bubonen auf, die allerdings auch fehlen können. Typisch bei einer Gonorrhö ist der schleimig-eitrige Ausfluss, beim Herpes ein kleinvesikulärer/-ulzeröser Befall meist kleiner als $5 \mathrm{~cm}$. Unspezifische Symptome macht die syphilitische Proktitis, die kaum Schmerzen, gelegentlich Tenesmen verursacht. Nach Nachweis des Erregers wird entsprechend antibiotisch behandelt. Bei einem LVG ist ein HIV-Test zwingend, denn einer Metaanalyse zufolge sind $75-100 \%$ dieser $\mathrm{Pa}$ tienten HIV-positiv. Um eine infektiöse Ursache nicht zu übersehen, empfahl Prof. Dr. Wolfgang Kruis, Köln, bei einer Proktitis, die auf eine antientzündliche Standardtherapie nicht anspricht, nach eingehender (Sexual-)Anamnese immer einen ausgedehnten Erregernachweis durchzuführen.

\section{Geburt, Trauma oder hohes Alter?}

\section{Therapie des Analsphinkterdefekts: Naht vor Schrittmacher}

Geburten, Traumata oder einfach nur das Alter erhöhen das Risiko für einen Analsphinkterdefekt. Dann ist chirurgisch die Analsphinkternaht als First-line-Therapie der sakralen Neuromodulation vorzuziehen.

Verglichen mit 20- bis 40-Jährigen sind bei 60- bis 80-Jährigen Ruhe- und Kontraktionsdruck reduziert. Laut PD Dr. A. J. Kroesen, Köln, sollte deshalb bei alten Men- schen vor einer tiefen Rektumresektion die Funktion des Analsphinkters geprüft und gegebenenfalls ein Stoma dem Kontinuitätserhalt vorgezogen werden.

Analsphinkterdefekte sind aber oft auch Folge eines Geburtstraumas. In einer aktuell publizierten Studie, die 3000 Frauen mit insgesamt 7000 Geburten auswertete, ließen sich bei 71-92\% der Frauen perineale Traumata feststellen, bei etwa einem Drittel einen zweitgradigen Dammriss und bei $5 \%$ eine Sphinkterruptur, erläuter-

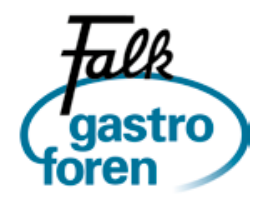

Besuchen Sie das nächste Falk Gastro Forum „Der akut erkrankte gastroenterologische Patient" am Samstag, den 06. Juli 2013 in Chemnitz; Infos: www.drfalkpharma.de/veranstaltungen

te Kroesen die Daten. Neben Biofeedback stehen dann therapeutisch die Analsphinkternaht und die sakrale Neuromodulation zur Verfügung. Mit der Analsphinkternaht konnte Kroesen nach eigenen Ergebnissen bei 35 Patientinnen eine Verbesserung des Holschneider-Scores von 3,2 präoperativ auf 12,4 postoperativ erreichen. In einem Vergleich von Sphinkternaht und sakraler Neuromodulation wurde eine ähnliche Kontinenzleistung erzielt. Laut Prof. Dr. Heinz Buhr, Berlin, ist die Sphinkternaht jedoch die erste Wahl. Sie könne für fünf bis zehn Jahre halten. Der Frau, für die die fehlende funktionelle Integrität des Beckenbodens ohnehin ein Problem ist, gibt dieses Vorgehen ein besseres Körpergefühlt, erläuterte er: „Einen Schrittmacher kann man später immer noch implantieren." (BF) 